\title{
Extract of Rhus verniciflua stokes protects against renal ischemia-reperfusion injury by enhancing Nrf2-mediated induction of antioxidant enzymes
}

\author{
DU RI CHOI ${ }^{1}$, JI HEUN JEONG ${ }^{1}$, KWANG-SIK YU ${ }^{1}$, NAM-SEOB LEE ${ }^{1}$, YOUNG-GIL JEONG ${ }^{1}$, \\ DO KYUNG KIM ${ }^{1}$, CHUN SOO NA $^{2}$, DAE SEUNG NA ${ }^{2}$, WON MIN HWANG ${ }^{3 *}$ and SEUNG-YUN HAN ${ }^{1,4^{*}}$ \\ ${ }^{1}$ Department of Anatomy, College of Medicine, Konyang University, Daejeon 302-718; \\ ${ }^{2}$ Lifetree Biotechnology Institute, Lifetree Biotech Co. Ltd, Suwon 441-813; ${ }^{3}$ Division of Nephrology, \\ Department of Internal Medicine, Konyang University Hospital; ${ }^{4}$ Myunggok Research Institute, College of Medicine, \\ Konyang University, Daejeon 302-718, Republic of South Korea
}

Received September 27, 2016; Accepted September 19, 2017

DOI: $10.3892 /$ etm.2018.5913

\begin{abstract}
Ischemia-reperfusion injury (IRI) may cause acute kidney disease (AKD) by mediating the oxidative stress-induced apoptosis of parenchymal cells. The extract of Rhus verniciflua Stokes (RVS) is used as a traditional herbal medicine as it exhibits anti-oxidant, anti-apoptotic and anti-inflammatory properties. Therefore, the current study investigated the therapeutic effect and the underlying mechanism of RVS on IRI-induced AKD in vivo and in vitro. The current study assessed the effects of RVS on a mouse model of renal IRI and in hypoxic human renal tubular epithelial HK-2 cells. The results demonstrated that the IRI-induced elevation of blood urea nitrogen, serum creatinine and lactate dehydrogenase was significantly attenuated by the intraoral administration of RVS (20 mg/kg/day) for 14 days prior to surgery. It was demonstrated that IRI surgery induced histological damage and cellular apoptosis in renal parenchyma, which were attenuated by pretreatment with RVS. Furthermore, in $\mathrm{HK}-2$ cells incubated with $300 \mu \mathrm{M} \mathrm{CoCl}_{2}$ to induce chemical hypoxia, it was demonstrated that RVS treatment significantly inhibited cell death and the production of reactive oxygen species (ROS). Furthermore, RVS treatment upregulated the
\end{abstract}

Correspondence to: Professor Won Min Hwang, Division of Nephrology, Department of Internal Medicine, Konyang University Hospital, 158 Gwanjeo-dong-ro, Seo, Daejeon 302-718, Republic of South Korea

E-mail: hwangwm@kyuh.ac.kr

Professor Seung-Yun Han, Department of Anatomy, College of Medicine, Konyang University, 158 Gwanjeo-dong-ro, Seo, Daejeon 302-718, Republic of South Korea

E-mail: jjzzy@konyang.ac.kr

${ }^{*}$ Contributed equally

Key words: ischemia-reperfusion injury, acute kidney disease, Rhus verniciflua Stokes, nuclear factor erythroid 2-related factor 2 levels of endogenous antioxidant enzymes, including heme oxygenase- 1 and catalase, as well as their upstream regulator nuclear factor erythroid 2-related factor 2, in HK-2 cells. Taken together, these results suggested that the intraoral administration of RVS induces a therapeutic effect on IRI-induced AKD. These effects are at least partly due to the attenuation of ROS production via upregulation of the antioxidant defense system in renal tubular cells.

\section{Introduction}

Ischemia-reperfusion injury (IRI) at least partly contributes to the high morbidity and mortality rates of patients with acute kidney injury (AKI) (1). A number of conditions, including kidney transplantation, induce renal IRI; this causes problems for many recipients of kidneys and may therefore negatively impact postoperative consequences $(2,3)$. Ischemic kidneys receive a reduced blood supply that does not meet metabolic demands, triggering severe hypoxia associated with renal tubular dysfunction $(4,5)$. Paradoxically, subsequent reperfusion fails to restore normal levels of perfusion; however, it induces further damage by activating detrimental mechanisms, such as those associated with oxidative stress (6).

Oxidative stress is a condition in which the formation of reactive oxygen species (ROS) exceeds the capacity of the endogenous antioxidant defense system $(7,8)$. In renal tissues, oxidative stress and associated sterile inflammation serve a major role in the pathogenesis of AKI, which occurs by complex mechanisms involving the ROS-mediated generation of vasoconstrictive prostanoids, lipid peroxidation and activation of pro-apoptotic factors (9-12). The increased accumulation of ROS and oxidative stress markers is frequently observed in the kidneys of animals with AKI (13). Under normal conditions, nuclear factor-erythroid-2 (NF-E2)-related factor 2 (Nrf2) serves a important role in combatting oxidative stress and the subsequent induction of $>250$ genes, including those encoding antioxidant enzymes (14). Attenuating the activation of Nrf2 may amplify cellular oxidative stress and associated pathological events. By contrast, strategies aimed at restoring 
Nrf2 activity may prevent the induction of IRI-associated renal injury and inhibit progression to AKI.

Despite important advances in understanding the pathological basis of AKI, clinical trials investigating the use of synthetic drugs to treat patients with AKI have been limited thus far (15). Considering the pathological features of AKI, including its rapid progression and involvement of multiple genes, it is understandable that researchers aim to develop multi-target, drug-based preventative strategies to treat this condition.

Traditional medicines often use multi-component extracts of natural products and may be developed as therapeutic strategies to treat AKI due to their multi-target potential and established biosafety (16). Phytochemicals from medicinal plants have attracted increased attention, as they are able to scavenge ROS and inhibit its formation, thereby reducing oxidative stress levels $(17,18)$. Rhus verniciflua Stokes (RVS) is a tree that belongs to the Anacardiaceae family that consists of $>250$ species primarily cultivated in Asian countries, including Korea and China (19). In Korea, RVS has been traditionally been added to chicken soup and used to treat conditions, including menstrual cycle irregularities, indigestion and high blood pressure (20). Several studies using nuclear magnetic resonance or liquid chromatography/mass spectrometry have revealed that many bioactive compounds exhibiting antioxidant activity are present in RVS, including gallic acid (GA), protocatechuic acid, quercetin, fustin, fisetin, sulfuretin, coumaric acid, kaempferol-3-O-glucoside, kaempferol and butein (21-25). Of these, fisetin has been reported to induce the expression of heme oxygenase-1 (HO-1), a major component of cellular antioxidant enzymes, in human umbilical vein endothelial cells by augmenting the nuclear translocation of Nrf2 (26). Furthermore, in a mouse model of inflammatory bowel disease it was demonstrated that GA activates and upregulates the expression of Nrf2 and associated antioxidant enzymes, including superoxide dismutase and catalase (CAT), thereby inducing a therapeutic effect (27). However, to date, no studies have been conducted to identify whether RVS itself can prevent the progression to AKI by activating the Nrf2-mediated defense mechanism against oxidative stress.

The aim of the current study was to investigate whether oral administration of RVS could prevent the progress of AKI via modulation of the Nrf2/antioxidant enzyme pathway, using in vivo and in vitro IRI-induced AKI models.

\section{Materials and methods}

Preparation of RVS extract. The RVS extract was supplied by Lifetree Biotech Co., Ltd. (Suwon, South Korea). The extract was prepared using a previously described protocol (28). Briefly, RVS timber was harvested in Wonju, (Gangwon-do, South Korea) and subsequently cut into pieces measuring $11 \times 1 \times 0.2 \mathrm{~cm}$. The pieces were mixed with water in a 1:10 w/v ratio and eluted with boiling water at $90-110^{\circ} \mathrm{C}$ for $4 \mathrm{~h}$. The extract was concentrated to yield a solid content of $15 \%$. Subsequently, the concentrated extract was diluted with an equal volume of dextrin and spray-dried. Urushiol, an allergenic constituent of RVS, was extracted. The urushiol-free RVS extract was authenticated by the Korea Advanced Food Research Institute (Seoul, South Korea).
Animals. A total of 32 8-week-old male C57BL/6 weighing 20-22 g were purchased from Samtako Bio Korea, Co., Ltd. (Osan, Korea). Mice were housed in an environmentally controlled room at $23 \pm 2^{\circ} \mathrm{C}$ at a relative humidity of $60 \pm 10 \%$ under a 12-h light/dark cycle. All mice had ad libitum access to water and food. Experiments were conducted in accordance with the 'Guide for the Care and Use of Laboratory Animals' (National Institutes of Health publication, 8th Edition, 2011) (29). All experiments involving mice were approved by the Institutional Animal Care and Use Committee (approved protocol number: P-16-10-A-02) of Konyang University (Daejeon, Korea).

Experimental design. Mice were randomly divided in 4 groups (all $\mathrm{n}=8$ ): A control group, an RVS-treated group (RVS), an IRI-operation group (IRI) and an IRI-operation group that received pretreatment with RVS (IRI+RVS). Whereas the control group was intraorally administered with $500 \mu \mathrm{l}$ of distilled water as a vehicle, the RVS group was intraorally administered with RVS (20 mg/kg/day) diluted in the same volume of vehicle over 14 days. For mice in the IRI+RVS group, the same dose of RVS as that administered to mice in the RVS group was applied prior to the IRI operation for the same duration. For mice in the IRI group, the same volume of distilled water was intraorally administered for 14 days prior to the IRI operation.

Operation and tissue sampling. Mice in the IRI and IRI+RVS groups were anesthetized with intraperitoneal injections of xylazine $(10 \mathrm{mg} / \mathrm{kg}$, Huons Global Co., Ltd., Seongnam, Korea) and ketamine (80 mg/kg, Bayer AG, Leverkusen, Germany). The abdominal area was sterilized with Betadine (Mundipharma International Ltd., Seoul, Korea) and a laparotomy was made by midline incision. To induce renal ischemia, bilateral renal pedicles were clamped for $60 \mathrm{~min}$ with serrated vascular clamps in a sterile operating field. Following removal of the clamp to allow reperfusion, the color of kidney was inspected to confirm the restoration of blood flow. The abdomen was then sutured in two layers. Following surgery, $50 \mathrm{ml} / \mathrm{kg}$ normal saline was administered intraperitoneally. Mice were allowed to recover in their home cages until they were fully awake and active. Following $23 \mathrm{~h}$ reperfusion, all mice were euthanized. The left kidneys of each group were removed and frozen for immunoblot assays and the right kidneys were fixed with formalin prior to histological study. Arterial blood was collected from the abdominal aorta for serological assays prior to euthanasia.

Serological assay. Following the collection of arterial blood, sera were obtained by centrifugation at a speed of $250 \mathrm{x} g$ for $10 \mathrm{~min}$ at $4^{\circ} \mathrm{C}$ and stored at $-70^{\circ} \mathrm{C}$ prior to assays. Levels of blood urea nitrogen (BUN; cat. no. 9903040; Fujifilm, Tokyo, Japan), creatinine (cat. no. 9903090; Fujifilm) and lactate dehydrogenase (LDH; cat. no. 9903190; Fujifilm) were measured through ELISA using a DRI-CHEM 3000 colorimetric analyzer (Fujifilm) at an excitation wavelength of 625 , 600 and $540 \mathrm{~nm}$, respectively.

Histology. Right kidney tissues were excised, fixed in $10 \%$ neutral buffered formalin for $48 \mathrm{~h}$ at $4^{\circ} \mathrm{C}$ and embedded in 
paraffin. Paraffin blocks were sliced into sections $5-\mu \mathrm{m}$ thick using the Leica RM2255 microtome (Leica Microsystems $\mathrm{GmbH}$, Wetzlar, Germany). Alterations in the histological structures of the kidney were examined by staining with hematoxylin and eosin (H-E). Two microscopic fields of tissue sections taken from every mouse were randomly selected. These fields were photographed at a magnification of $x 400$ using a digital camera connected to a Leica DM4 light microscope (Leica Microsystems $\mathrm{GmbH}$ ) and examined by three blinded observers. Renal tubular injury was scored by estimating the percentage of tubules in the corticomedullary junction and outer medulla that exhibited epithelial necrosis or necrotic debris and determined as follows: 0 , none; $1+,<10 ; 2+$, 10-25; 3+, 26-45; 4+, 46-75; and 5+, >75\%.

Terminal deoxynucleotidyl transferase dUTP nick end labeling (TUNEL) assay. Paraffin-embedded kidney sections were deparaffinized in xylene and rehydrated in a graded series of ethanol solutions. The TUNEL assay was performed using an In Situ Cell Death Detection kit (Roche Diagnostics, Indianapolis, IN, USA), following the manufacturer's protocol. Nuclei were counterstained with DAPI. Among the resulting TUNEL-stained tissue sections, two sections from each mouse were randomly selected and captured using a digital camera connected to a Leica DMI6000 inverted microscope (Leica Microsystems) to detect fluorescence. Images were visualized at a magnification of $x 400$. The number of TUNEL-positive stained nuclei per high power field (HPF) was counted and averaged by three blind observers.

Cell culture and in vitro hypoxia. Human kidney epithelial cells, HK-2 cells, were obtained from the Korean Cell Line Bank (Seoul, Korea). Cells were cultured for $72 \mathrm{~h}$ in $25-\mathrm{cm}^{2}$ cell culture flasks containing RPMI 1640 culture medium supplemented with $10 \%$ fetal bovine serum, $100 \mathrm{U} / \mathrm{ml}$ penicillin and $100 \mathrm{mg} / \mathrm{ml}$ streptomycin at $37^{\circ} \mathrm{C}$ in a humidified atmosphere consisting of $5 \% \mathrm{CO}_{2} / 95 \%$ air. All of the aforementioned chemicals were purchased from Thermo Fisher Scientific, Inc. (Waltham, MA, USA). For in vitro hypoxia conditioning, cobalt chloride $\left(\mathrm{CoCl}_{2}\right)$, a chemical inducer of hypoxia-inducible factor-1, was used. To determine the approximate values of the median lethal dose (LD50) and the median lethal time (LT50) of $\mathrm{CoCl}_{2}$, dose-dependent and time-dependent changes in the viability of HK-2 cells following treatment with different doses $(0,10,100,300,600$ and $900 \mu \mathrm{l})$ of $\mathrm{CoCl}_{2}$ for various incubation times $(0,6,12$, 24 and $48 \mathrm{~h}$ ) were analyzed. The LD50 and LT50 of $\mathrm{CoCl}_{2}$ were $300 \mu \mathrm{M}$ and $24 \mathrm{~h}$, respectively; this dose of $\mathrm{CoCl}_{2}$ and incubation time was used in all subsequent experiments, apart from the ROS measurement, in which 30 min was selected as the incubation time.

Cell viability assay. HK-2 cells were seeded in a 96-well plate at a density of $1 \times 10^{4}$ cells/well. After incubation with RVS $(0$, $40,80,120,160$ and $200 \mu \mathrm{g} / \mathrm{ml}$ ) with or without $300 \mu \mathrm{M} \mathrm{CoCl}_{2}$ for $24 \mathrm{~h}$, cell viability was measured by estimating the amount of reduced 3-(4,5-dimethyl-2-thiazolyl)-2,5-diphenyltetrazolium bromide (MTT), a pale yellow substrate that is reduced by mitochondrial activity in living cells, following exposure to different conditions. For this, $100 \mu \mathrm{l}$ culture medium was aspirated and $20 \mu \mathrm{l}$ MTT (Sigma-Aldrich; Merck KGaA, Darmstadt, Germany) diluted in PBS at a ratio of $5 \mathrm{mg} / \mathrm{ml}$ was added to the wells and incubated for $4 \mathrm{~h}$ at $37^{\circ} \mathrm{C}$. The resulting formazan crystals were dissolved following the addition of dimethyl sulfoxide. Using an Epoch microplate spectrophotometer (BioTek Instruments, Inc., Winooski, VT, USA), cell viability was estimated by detecting color intensity at an excitation wavelength of $570 \mathrm{~nm}$.

ROS measurement. HK-2 cells grown on glass coverslips were split into the following 4 groups, with 5 samples per group $(n=5)$ : A control group (cells treated with distilled water), an RVS group (cells treated with $40 \mu \mathrm{g} / \mathrm{ml} \mathrm{RVS}$ ), a $\mathrm{CoCl}_{2}$ group (cells treated with $300 \mu \mathrm{M} \mathrm{CoCl}_{2}$ ) and a $\mathrm{CoCl}_{2}+\mathrm{RVS}$ group (cells treated with $40 \mu \mathrm{g} / \mathrm{ml} \mathrm{RVS} \mathrm{and} 300 \mu \mathrm{M} \mathrm{CoCl}_{2}$ ). Distilled water and RVS were diluted in culture medium and applied to the cells for $24 \mathrm{~h}$. $\mathrm{CoCl}_{2}$ was subsequently added to the culture medium for $30 \mathrm{~min}$. Subsequently, the amount of cellular ROS was measured using the OxiSelect ${ }^{\mathrm{TM}}$ Intracellular ROS assay kit (Cell Biolabs Inc., San Diego, CA, USA) following the manufacturer's protocol. 2',7'-dichlorodihydrofluorescin diacetate (DCFH-DA) is converted to 2',7'-dichlorodihydrofluorescin (DCFH), which is rapidly oxidized to fluorescent 2',7'-dichlorodihydrofluorescein (DCF) by ROS. The fluorescence intensity is proportional to ROS levels within the cell cytosol. From each group, $\geq 5 \mathrm{HPFs}$ were examined using a fluorescence microscope connected to a digital camera at a magnification of $x 400$. The fluorescent intensities in each HPF were measured and averaged using an image analysis system (ImageJ software; version, 1.49; National Institutes of Health, Bethesda, MD, USA).

Immunofluorescence. $\mathrm{HK}-2$ cell groups (control, RVS, $\mathrm{CoCl}_{2}$ and $\mathrm{CoCl}_{2}+\mathrm{RVS}$ ) were grown on glass coverslips $(\mathrm{n}=5$ per group). Distilled water and RVS were diluted in culture medium and applied to the cells for $24 \mathrm{~h}$. $\mathrm{CoCl}_{2}$ was subsequently added to the culture medium for $24 \mathrm{~h}$. Coverslips were fixed with $4 \%$ paraformaldehyde for $30 \mathrm{~min}$ at $4^{\circ} \mathrm{C}$, blocked with $1 \%$ bovine serum albumin (Sigma-Aldrich; Merck KGaA) diluted in PBS for $1 \mathrm{~h}$ at $24^{\circ} \mathrm{C}$ and incubated with anti-Nrf2 rabbit polyclonal antibody (1:1,000, cat. no., sc-722; Santa Cruz Biotechnology, Dallas, TX, USA) for $24 \mathrm{~h}$ at $4^{\circ} \mathrm{C}$. Subsequently, cells were incubated with Alexa Fluor ${ }^{\circledR}$ 488-conjugated goat anti-rabbit antibody (1:2,000, cat. no., ab150077; Abcam, Cambridge, UK) at room temperature for $1 \mathrm{~h}$. Coverslips were then counterstained for 5 min with DAPI and mounted with ProLong Gold mounting media (cat. no., 8961; Cell Signaling Technology, Danvers, MA, USA) at $24^{\circ} \mathrm{C}$ and examined using the LSM 700 laser scanning confocal microscope (magnification, $\mathrm{x} 400$; Zeiss AG, Oberkochen, Germany).

Western blot analysis. Total proteins were extracted from the HK-2 cell groups. Total protein extraction and western blot analyses were performed as previously described (15). Cells were harvested, transferred into lysis buffer (Pro-Prep ${ }^{\mathrm{TM}}$; intron Biotechnology, Inc., Seoungnam, Korea) and homogenized. The total protein concentration of the supernatant was determined using a BCA protein assay (Pierce Biotechnology, Inc., Rockford, IL, USA). Subsequently, the protein sample $(30 \mu \mathrm{g} / \mathrm{ml})$ was separated by $10 \%$ SDS-PAGE and transferred 

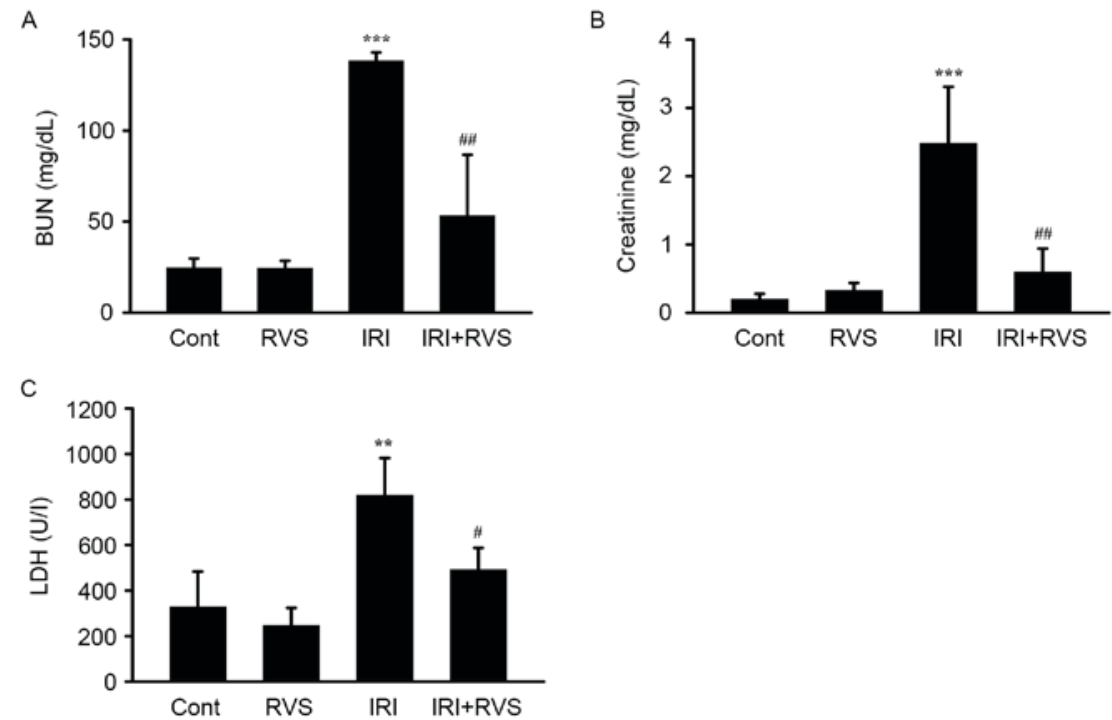

Figure 1. Effect of RVS on IRI-induced alterations on serum levels of renal function parameters. Effects of RVS ( $20 \mathrm{mg} / \mathrm{kg} / \mathrm{day})$ intraoral administration for 14 days on (A) serum BUN, (B) creatinine and (C) LDH levels in mice with or without IRI ( $n=7)$. Values are expressed as the mean \pm standard error of the mean ${ }^{* *} \mathrm{P}<0.01$ and ${ }^{* * *} \mathrm{P}<0.001$ vs. Cont group; ${ }^{*} \mathrm{P}<0.05$ and ${ }^{\# \#} \mathrm{P}<0.01$ vs. the IRI group. RVS, Rhus verniciflua Stokes; IRI, ischemia-reperfusion injury; Cont, control; BUN, blood urea nitrogen; LDH, lactate dehydrogenase.

onto a polyvinylidenedifluoride (PVDF) membrane (Bio-Rad Laboratories, Inc., Hercules, CA, USA), which was blocked with $5 \%$ nonfat milk in Tris-buffered saline with $0.1 \%$ Tween 20 (TBS-T) for $2 \mathrm{~h}$ at room temperature. The membranes were incubated with rabbit polyclonal antibodies against HO-1 (1:1,000, cat. no. sc-136960), CAT (1:500, cat. no. sc-271803) and GAPDH (1:2,000, cat. no. sc-25778) for $24 \mathrm{~h}$ at $4^{\circ} \mathrm{C}$. Proteins were then incubated with horseradish peroxide-conjugated anti-rabbit immunoglobulin $\mathrm{G}(1: 1,000$, cat. no. sc-2030) for $1 \mathrm{~h}$ at $36^{\circ} \mathrm{C}$. To quantify the nuclear translocation of $\mathrm{Nrf} 2$, a subcellular protein fractionation kit (cat. no. 87790; Thermo Fisher Scientific, Inc.) was used to isolate nuclear fractions following the manufacturer's protocol. Protocols for the determination of total protein concentration in nuclear fractions, their SDS-PAGE separation and their transfer to PVDF membranes were identical as those aforementioned. The membranes were incubated with the Nrf2 rabbit polyclonal antibody (1:1,000; cat. no. sc-722) for $24 \mathrm{~h}$ at $4^{\circ} \mathrm{C}$. Proteins were then incubated with horseradish peroxide-conjugated anti-rabbit immunoglobulin G (1:1,000; cat. no., sc-2030) for $1 \mathrm{~h}$ at $36^{\circ} \mathrm{C}$. The quality of the isolation of nuclear fractions was confirmed by immunoblots using Lamin B rabbit polyclonal antibody (1:1,000; cat. no. sc-3755). All aforementioned antibodies were purchased from Santa Cruz Biotechnology (Dallas, TX, USA). Following five washes with PBS, proteins on the PVDF membranes were detected using a chemiluminescence detection system (Amersham ECL Prime Western Blotting Detection Reagent; GE Healthcare Life Sciences, Little Chalfont, UK) following the manufacturer's protocol. The resulting bands were photographed using a Davinch-Chemi imaging device (Davinch-K, Seoul, Korea) and their intensities were quantified using ImageJ (version, 1.49; National Institutes of Health, Bethesda, MD, USA).

Statistical analysis. All data are presented as the mean \pm standard error of the mean. Statistical analysis was conducted using PASW version 18 (SPSS, Inc., Chicago, IL, USA). Comparisons of data from different groups were performed with one-way analysis of variance followed by a Tukey post-hoc test. $\mathrm{P}<0.05$ was considered to indicate a statistically significant difference. Each ' $n$ ' value refers to the number of separate experiments conducted.

\section{Results}

RVS attenuates the IRI-induced decrease of renal function parameters. To clarify the role of RVS in renal IRI, C57BL/6 mice were subjected to IRI ( $1 \mathrm{~h}$ renal artery occlusion followed by $23 \mathrm{~h}$ reperfusion) with or without RVS pretreatment (14 days, $20 \mathrm{mg} / \mathrm{kg} /$ day). RVS alone did not alter renal function, as indicated by the unchanged levels of serum BUN, creatinine and LDH in the RVS group (Fig. 1). By contrast, compared with the control group, the IRI group clearly exhibited AKI and renal dysfunction, as indicated by significant increases in levels of serum BUN $(138.3 \pm 4.5$ vs. $24.6 \pm 5.1, \mathrm{P}<0.001)$, creatinine $(2.5 \pm 0.8$ vs. $0.16 \pm 0.05, \mathrm{P}<0.001)$ and $\mathrm{LDH}(818.8 \pm 162.5$ vs. $317.8 \pm 129.5, \mathrm{P}<0.01)$. However, there were significant reductions in serum $\mathrm{BUN}(53.2 \pm 33.5$ vs. $138.3 \pm 4.5, \mathrm{P}<0.01)$, creatinine $(0.6 \pm 0.3$ vs. $2.5 \pm 0.8, \mathrm{P}<0.01)$ and $\mathrm{LDH}(491.7 \pm 95.2$ vs. $818.8 \pm 162.5, \mathrm{P}<0.05)$ levels compared with those in the IRI group. These data indicate that RVS may prevent the IRI-induced deterioration of renal function.

RVSattenuates IRI-induced histological damage. Representative images of H-E stained tissue sections of the corticomedullary junction (Fig. 2A) and outer medulla (Fig. 2B) indicate that renal tissue from the control and RVS groups exhibited normal morphology. Kidneys in the IRI group exhibited acute tubular damage, indicated by tubular swelling, infiltrated inflammatory cells (arrowhead) and loss of the brush border due to apoptosis/necrosis of the tubular epithelium (arrow) in renal tissue. However, RVS treatment markedly attenuated these 


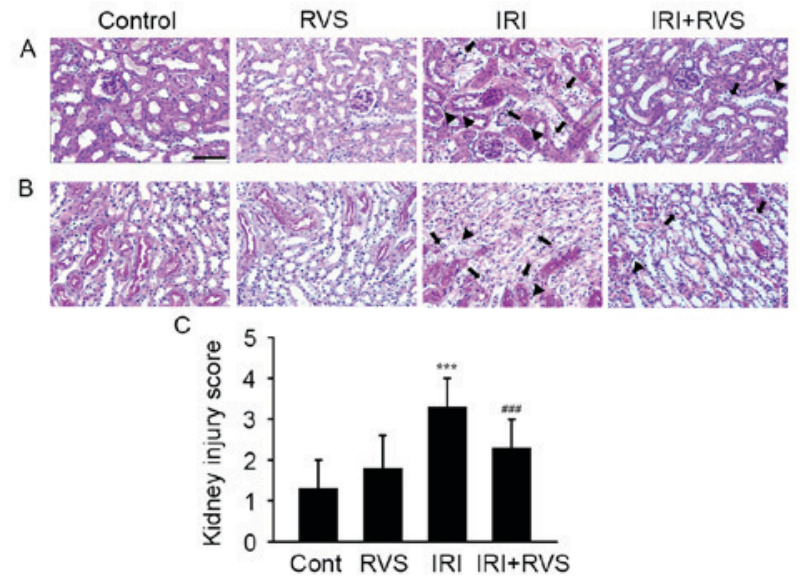

Figure 2. Effect of RVS on IRI-induced alterations on renal histoarchitecture. RVS $(20 \mathrm{mg} / \mathrm{kg} / \mathrm{day})$ was intraorally administered for 14 days to mice with or without IRI and their kidneys were stained with hematoxylin and eosin for histological examination. Representative images of (A) the corticomedullary junction and (B) the outer medulla of Cont, RVS, IRI and IRI+RVS groups ( $\mathrm{n}=7$ per group) are presented. Black arrowheads and black arrows indicate an infiltrated inflammatory cells and necrotic tubules, respectively. Scale bar $=200 \mu \mathrm{m}$. (C) Renal tubular injury was scored by estimating the percentage of tubules in the corticomedullary junction and outer medulla that exhibited epithelial necrosis or had necrotic debris and were cast as follows: 0 , none; $1+,<10 ; 2+, 10-25 ; 3+, 26-45 ; 4+, 46-75$; and $5+,>75 \%$. Values are expressed as the mean \pm standard error of the mean. ${ }^{* * *} \mathrm{P}<0.001$ vs. Cont group; ${ }^{\# \# \# / P}<0.001$ vs. IRI group. RVS, Rhus verniciflua Stokes; IRI, ischemia-reperfusion injury; Cont, control.

pathological features, suggesting that RVS protects the tubular epithelium. This hypothesis is supported by the fact that kidney injury scores were significantly lower in the IRI+RVS group compared with the IRI group $(3.3 \pm 0.7$ vs. $2.3 \pm 0.7$, $\mathrm{P}<0.001$; Fig. 2C).

RVS inhibits renal tubular cell apoptosis. Tubular apoptosis normally precedes/accompanies tubular cell death; therefore, the number of apoptotic cells in the kidney was quantified using a TUNEL assay. As presented in a representative image (Fig. 3A) and in a graph presenting quantified results (Fig. 3B), the number of TUNEL-positive cells was significantly increased in the IRI group compared with the control group (58.3 \pm 11.0 vs. $4.0 \pm 2.0, \mathrm{P}<0.001)$. However, significantly fewer TUNEL-positive cells were visible in the IRI+RVS group than in the IRI group $(12.7 \pm 2.5$ vs. $58.3 \pm 11.0, \mathrm{P}<0.001)$. As expected, the number of TUNEL-positive cells in the RVS group did not differ significantly compared with the control group. Collectively, these data suggest that RVS protects the kidney from the histological deterioration of renal tubules and that this effect was due to the inhibition of apoptosis in the renal tubular epithelium.

RVS protects $\mathrm{HK}-2$ cells from $\mathrm{CoCl}_{2}$-induced damage. To confirm the protective effect of RVS on cell damage caused by IRI, hypoxia was chemically induced by the addition of $\mathrm{CoCl}_{2}$, a well-known inducer of in vitro IRI, in the human kidney epithelial cell line HK-2. The approximate LD50 and LT50 values of $\mathrm{CoCl}_{2}$ were identified as $300 \mu \mathrm{M}$ and $24 \mathrm{~h}$, respectively (rectangular boxes; Fig. 4A and B). There was no significant decrease in cell viability of HK-2 cells incubated with different doses $(0-200 \mu \mathrm{g} / \mathrm{ml})$ of RVS, indicating that RVS does not induce

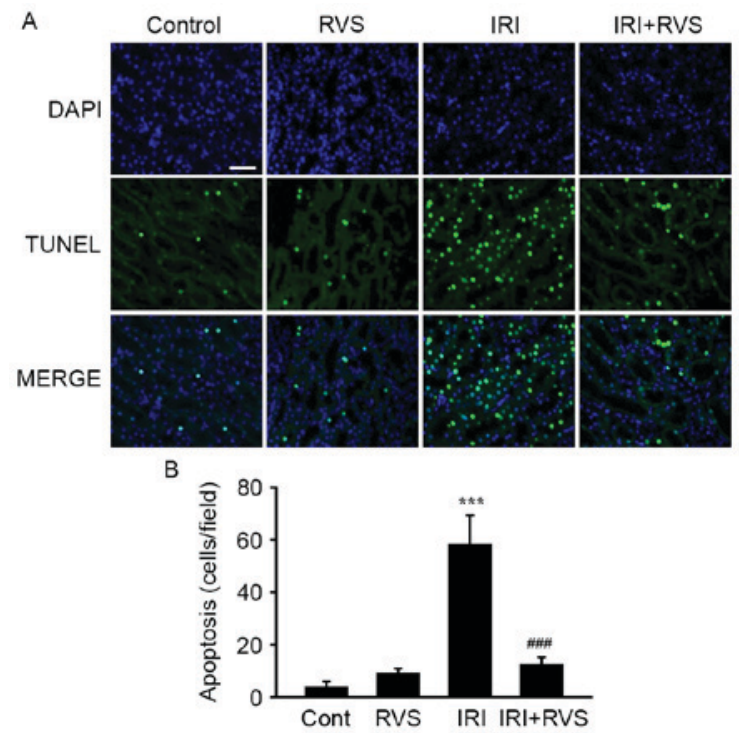

Figure 3. Effect of RVS on IRI-induced apoptosis of tubular epithelium $\operatorname{RVS}(20 \mathrm{mg} / \mathrm{kg} / \mathrm{day})$ was intraorally administered for 14 days to mice with or without IRI and their kidneys were processed for TUNEL-staining. (A) Representative fluorescence images of the outer medulla of the Cont, RVS, IRI and IRI+RVS groups ( $n=7$ per group) indicate the TUNEL-stained nuclei (green) of apoptotic tubular epithelium. DAPI was used to counterstain the nuclei (blue). Scale bar $=100 \mu \mathrm{m}$. (B) The number of apoptotic tubular epithelial cells in 1 HPF was counted and averaged. Two sections of each mouse (14 sections per group) were randomly selected and $\geq 5$ HPF were examined for each section. Values are expressed as the mean \pm standard error of the mean. ${ }^{* * * *} \mathrm{P}<0.001$ vs. Cont; ${ }^{\# \# \#} \mathrm{P}<0.001$ vs. IRI group. RVS, Rhus verniciflua Stokes; IRI, ischemia-reperfusion injury; Cont, control; HPF, high power field.

toxicity in this cell line (Fig. 4C). Notably, incubation with 40-160 RVS $\mu \mathrm{g} / \mathrm{ml}$ significantly increased the viability of HK-2 cells treated with $300 \mu \mathrm{M} \mathrm{CoCl}_{2}$ for $24 \mathrm{~h}(\mathrm{P}<0.01$; Fig. 4D). Collectively, these results demonstrate that pre-treatment with RVS inhibits the cytotoxic effect of $\mathrm{CoCl}_{2}$ on HK-2 cells.

RVS inhibits $\mathrm{CoCl}_{2}$-induced ROS generation in $\mathrm{HK}-2$ cells. To determine whether antioxidant properties are involved in the protective effect of RVS against $\mathrm{CoCl}_{2}$-induced damage in HK-2 cells, intracellular ROS levels in different cell groups was detected using DCFH-DA. As RVS significantly elevated cell viability even at a dose of $40 \mu \mathrm{g} / \mathrm{ml}$, this dose was used in the current study. As indicated in a representative fluorescence image (Fig. 5A) and a quantitative graph (Fig. 5B), cells treated with $40 \mu \mathrm{g} / \mathrm{ml}$ RVS for $24 \mathrm{~h}$ exhibited significantly higher ROS intensity compared with those in the control group $(3.9 \pm 1.3$ vs. $1.0 \pm 0, \mathrm{P}<0.05)$. Cells incubated with $300 \mu \mathrm{M} \mathrm{CoCl}_{2}$ for 30 min exhibited an even higher ROS intensity compared with the control $(21.4 \pm 5.9, \mathrm{P}<0.001$ vs. control). However, cells treated with $40 \mu \mathrm{g} / \mathrm{ml} \mathrm{RVS}$ for $24 \mathrm{~h}$ and then incubated with $300 \mu \mathrm{M} \mathrm{CoCl}_{2}$ for 30 min exhibited significantly lower ROS intensity than the $\mathrm{CoCl}_{2}$ group (6.0 \pm 1.4 vs. $\left.21.4 \pm 5.9, \mathrm{P}<0.001\right)$. These data suggest that the RVS-mediated protection of HK-2 cells from $\mathrm{CoCl}_{2}$ is at least partly due to the inhibition of ROS generation.

RVS increases the nuclear translocation of $\mathrm{Nrf} 2$ and expression of HO-1 and CAT. To determine the upstream signaling pathway involved in the RVS-mediated inhibition 

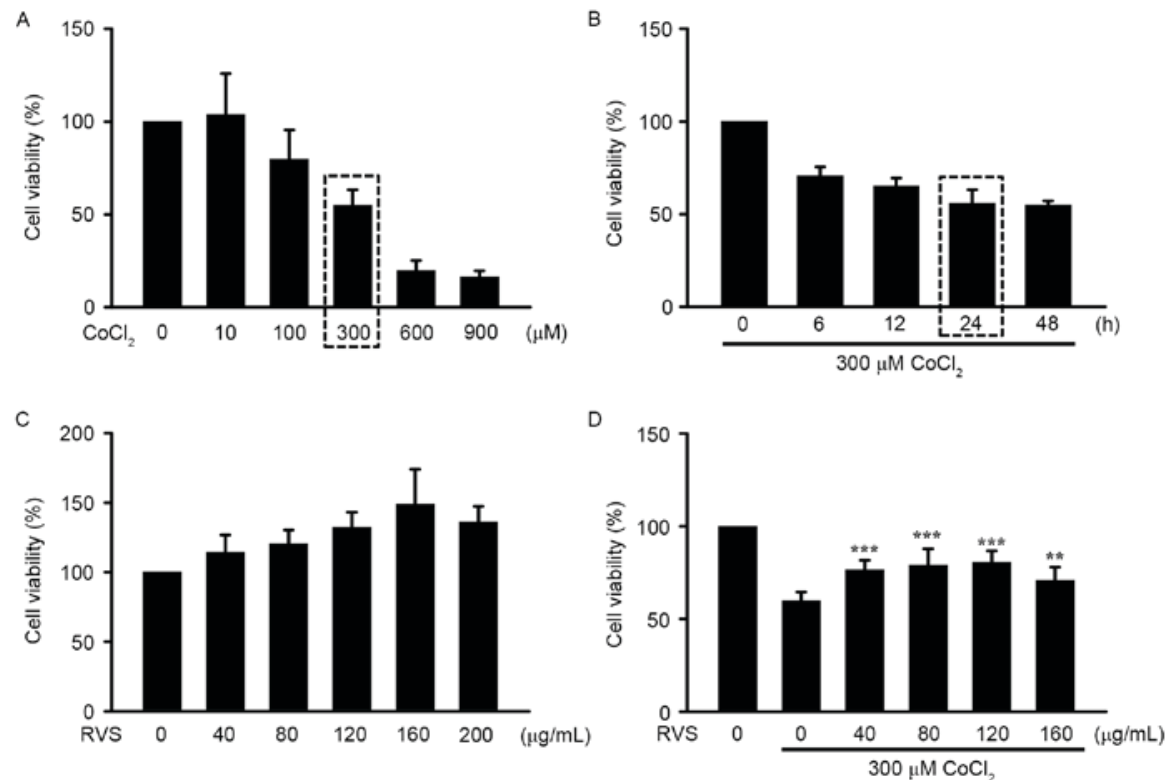

Figure 4. Effects of RVS on HK-2 cell viability in vitro following IRI. HK-2 cells were pretreated with or without RVS for $24 \mathrm{~h}$ and then challenged with CoCl $\mathrm{C}_{2}$ a chemical known to imitate hypoxic injury. Cell viability was determined using an MTT assay. (A) Dose-dependent cell viability following incubation with varying concentrations of $\mathrm{CoCl}_{2}(0,10,100,300,600$ and $900 \mu \mathrm{M})$ and (B) time-dependent cell viability following incubation with $300 \mu \mathrm{M} \mathrm{CoCl}{ }_{2}$ for different durations $(0,6,12,24$ and $48 \mathrm{~h})$. (C) Identification of the safe concentration range of RVS by incubating cells with different concentrations of RVS ( $0,40,80$, $120,160$ and $200 \mu \mathrm{g} / \mathrm{ml})$. (D) The effects of $24 \mathrm{~h}$ incubation with RVS $(0,40,80,120$ and $160 \mu \mathrm{g} / \mathrm{ml})$ and on $\mathrm{CoCl}_{2}(300 \mu \mathrm{M})$-induced alteration of $\mathrm{HK}-2$ cell viability were assessed. Values are presented as the mean \pm standard error of the mean. ${ }^{* *} \mathrm{P}<0.01$ and ${ }^{* * * *} \mathrm{P}<0.001 \mathrm{vs}$. cells incubated with $300 \mu \mathrm{M} \mathrm{CoCl}{ }_{2}$ alone. RVS, Rhus verniciflua Stokes; IRI, ischemia-reperfusion injury.
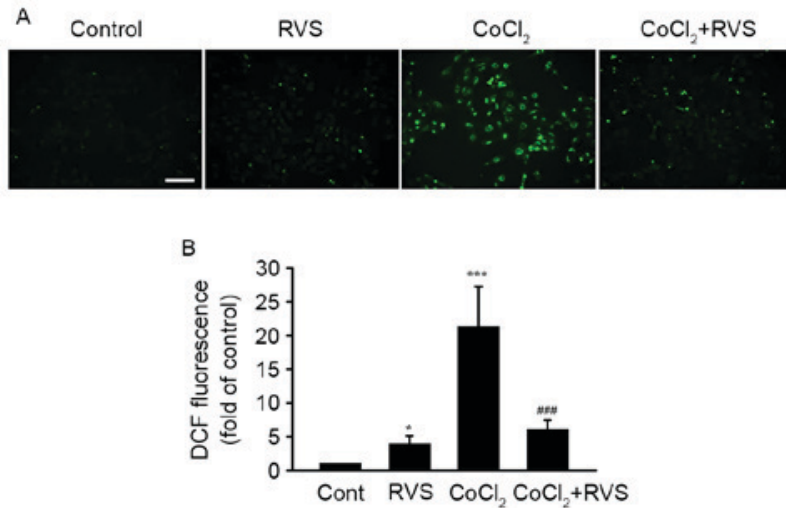

Figure 5. RVS inhibits $\mathrm{CoCl}_{2}$-induced ROS production in HK-2 cells. HK-2 cells were pretreated with $40 \mu \mathrm{g} / \mathrm{ml} \mathrm{RVS}$ for $24 \mathrm{~h}$ and incubated with or without $\mathrm{CoCl}_{2}$ for $30 \mathrm{~min}$. Intracellular ROS levels were determined by DCF fluorescence. DCF fluorescence results from the oxidation of DCF-DA by ROS were measured. (A) Representative images and (B) quantification of $\mathrm{CoCl}_{2}$-induced DCF fluorescence are presented. Scale bar=100 $\mu \mathrm{m}$. In the graph, DCF fluorescence of each group is expressed as mean \pm standard error of the mean. $\mathrm{n}=5$ per group. ${ }^{*} \mathrm{P}<0.05$ and ${ }^{* * * *} \mathrm{P}<0.001$ vs. Cont group and

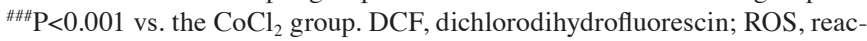
tive oxygen species; RVS, Rhus verniciflua Stokes; IRI, ischemia-reperfusion injury; Cont, control.

of ROS generation, the nuclear translocation of Nrf2 and the expression of cellular antioxidant enzymes HO-1 and CAT were assessed, which are encoded by Nrf2 target genes. The translocation of Nrf2 from the cytosol into the nucleus was assessed using confocal microscopy. The results indicated that Nrf2 proteins (green) were primarily distributed in the cytoplasm of the control group (Fig. 6). In the RVS group $(40 \mu \mathrm{g} / \mathrm{ml}, 24 \mathrm{~h})$, the majority of the cytoplasmic Nrf2 was

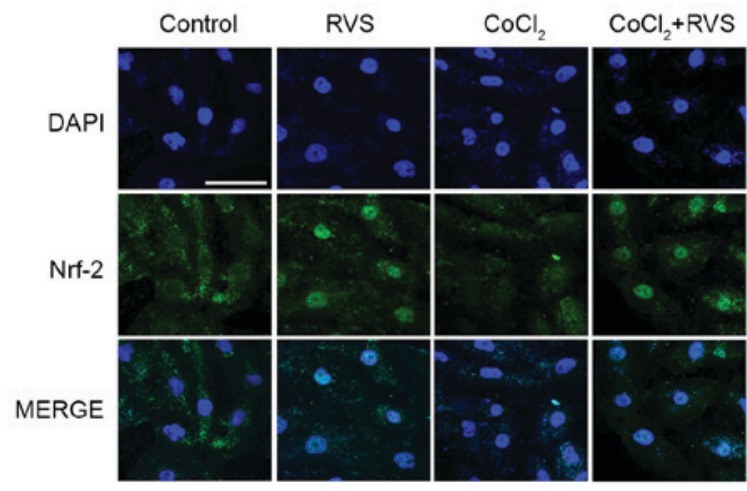

Figure 6. Effect of RVS on the nuclear translocation of Nrf2. Cells were pretreated with or without $40 \mu \mathrm{g} / \mathrm{ml} \mathrm{RVS}$ for $24 \mathrm{~h}$ and incubated for a further $24 \mathrm{~h}$ with or without $\mathrm{CoCl}_{2}$. Cells were fixed and immune-stained with an anti-Nrf2 antibody for $2 \mathrm{~h}$. Nuclei were counter-stained with DAPI and the images were captured by confocal microscopy. Scale bar $=100 \mu \mathrm{m}$. RVS , Rhus verniciflua Stokes; Nrf2, nuclear factor-erythroid-2-related factor 2.

translocated into the nucleus, as indicated by intense nuclear $\mathrm{Nrf} 2$ staining in the immunofluorescence assay. In the $\mathrm{CoCl}_{2}$ group (300 $\mu \mathrm{M}, 30 \mathrm{~min}), \mathrm{Nrf} 2$ fluorescence was decreased compared with that of control group and fluorescence was mostly cytosolic. However, robust nuclear translocation of $\mathrm{Nrf} 2$ was detected in the $\mathrm{CoCl}_{2}+\mathrm{RVS}$ group.

To confirm the translocation of $\mathrm{Nrf} 2$ into the nucleus following RVS treatment with or without $\mathrm{CoCl}_{2}$, cell lysates were separated into cytosolic and nucleic fractions and the expression of Nrf 2 in each fraction was measured using western blot analysis. Representative band images (Fig. 7A) and quantification of the results (Fig. 7B) demonstrated that Nrf2 expression was significantly increased in the nuclear fraction of the RVS 

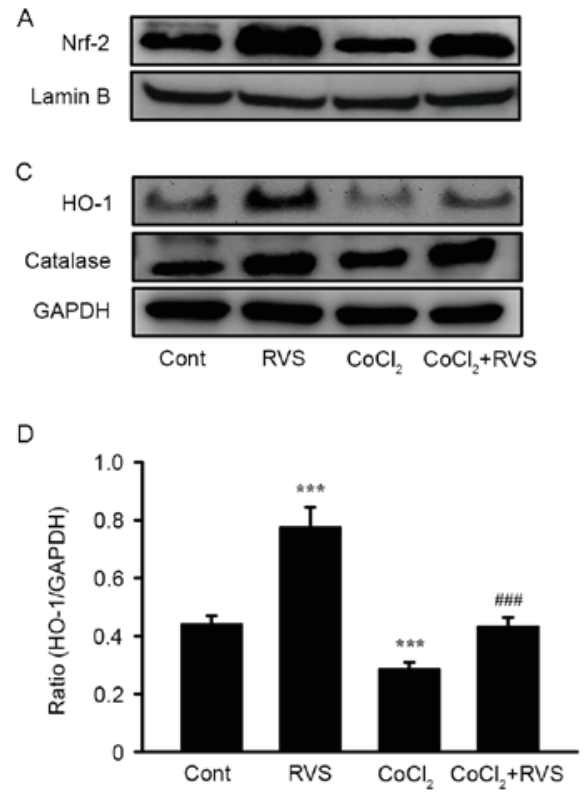

B

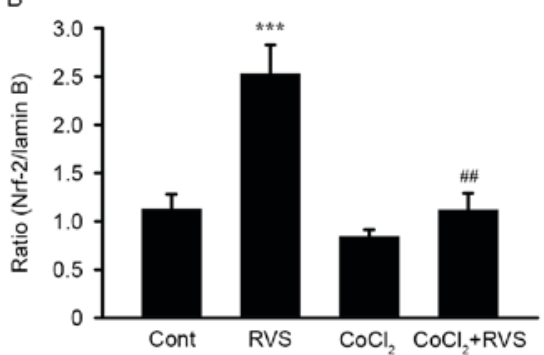

E

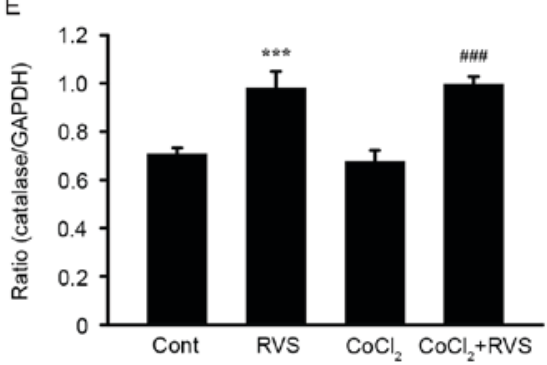

Figure 7. Effect of RVS on Nrf2, HO-1 and CAT expression in cells with or without treatment with $\mathrm{CoCl}_{2}$. Nuclear fractions were prepared and analyzed by western blot analysis with the Nrf2 antibody. (A) Representative immunoblots of Nrf2 expression. (B) Band densities of nuclear Nrf2 were converted to fold change to that of the control group. Lamin B, a nuclear protein, was used as a loading control. (C) Representative western blot images representing the expression of HO-1 and CAT were obtained using the corresponding antibodies. Band densities of (D) HO-1 and (E) CAT expression were converted to the fold change to that of the control group. GAPDH was used as a loading control. $\mathrm{n}=5$. Values are presented as the mean \pm standard error of the mean. ${ }^{* *} \mathrm{P}<0.01$ and ${ }^{* * *} \mathrm{P}<0.001$ vs. Cont group; ${ }^{\# \#} \mathrm{P}<0.001$ vs. the $\mathrm{CoCl}_{2}$ group. Cont, control; RVS, Rhus verniciflua Stokes; IRI, ischemia-reperfusion injury; Nrf2, nuclear factor-erythroid-2-related factor 2; HO-1, heme oxygenase-1; CAT, catalase.

group compared with the control group $(2.5 \pm 0.3$ vs. $1.1 \pm 0.2$ $\mathrm{P}<0.001)$. As expected, compared with the control group, the $\mathrm{CoCl}_{2}$ group exhibited a reduction in the expression of nuclear Nrf2 (0.8 \pm 0.1 vs. $1.1 \pm 0.2, \mathrm{P}<0.01)$. However, Nrf2 expression was significantly increased in the $\mathrm{CoCl}_{2}+\mathrm{RVS}$ group compared with the $\mathrm{CoCl}_{2}$ group (1.2 \pm 0.3 vs. $\left.0.8 \pm 0.1, \mathrm{P}<0.01\right)$.

Subsequently, the expression of HO-1 and CAT in the cell homogenates from each treatment group was measured. These are major cellular antioxidant enzymes that are upregulated following Nrf2 translocation. As presented in the representative band images (Fig. 7C) and quantification graphs for $\mathrm{HO}-1$ (Fig. 7D) and CAT (Fig. 7E), levels of HO-1 and CAT were significantly increased in cell homogenates from the RVS group compared with those of the control group $(0.8 \pm 0.1$ vs. $0.44 \pm 0.03$ and $1.0 \pm 0.06$ vs. $0.7 \pm 0.02$, respectively, both $\mathrm{P}<0.001)$. In the $\mathrm{CoCl}_{2}$ group, levels of $\mathrm{HO}-1$ were significantly decreased $(\mathrm{P}<0.001)$ but levels of CAT were unchanged, compared with the control group. However, levels of HO-1 and CAT were significantly increased in the $\mathrm{CoCl}_{2}+\mathrm{RVS}$ group compared with the $\mathrm{CoCl}_{2}$ group $(0.43 \pm 0.03$ vs. $0.28 \pm 0.02$ and $1.0 \pm 0.03$ vs. $0.7 \pm 0.04$, respectively, both $\mathrm{P}<0.001$ ). Collectively, these data suggest that RVS exerts antioxidant effects by increasing the nuclear translocation of $\mathrm{Nrf} 2$ and thereby upregulating the expression of cellular antioxidant enzymes, including HO-1 and CAT.

\section{Discussion}

ROS are continuously generated in live cells as a natural product of oxidative metabolism and act as messengers for signal transduction in various cellular processes, including mitosis, gene expression and proliferation (30-33). However, excess
ROS are implicated in various pathologies including ageing, cancer, inflammation and renal disease (34-37). Consequently, antioxidants with the potential to reduce ROS may prevent or treat diseases associated with oxidative injury (38). Therefore, various natural products with ROS-scavenging properties may be developed as promising therapeutic strategies to treat oxidative damage-related conditions. Exposure to certain natural products elevates intracellular ROS levels and alleviates mild oxidative stress. Such mild oxidative stress may initiate the signal transduction responsible for the induction and activation of antioxidant enzymes (39). In accordance with this hypothesis, the results of the current study indicate that treatment with RVS induces a significant, elevation of ROS in HK-2 cells. Thus, RVS may increase the expression of HO-1 and CAT by inducing mild oxidative stress.

The current study focused on the modulatory effects of RVS on Nrf2, a redox-sensitive transcription factor, as an upstream regulator of HO-1 and CAT (40). Nrf2 is a member of the NF-E2 family of basic leucine zippers and is able to deactivate ROS (41). Under normal conditions, Nrf2 is located in the cytoplasm and is sequestered by Kelch-like ECH-associated protein 1 (Keap1), which disturbs the nuclear translocation of Nrf2 (42). Following activation of the Nrf2/antioxidant responsive element (ARE) pathway, the expression of various antioxidant enzymes, including $\mathrm{HO}-1$ and CAT, are induced (43). Thus, there is substantial interest in therapeutic candidates that may augment this pathway. To date, in vitro and in vivo experiments have proven that phytochemicals, including curcumin, epigallocatechin-3-gallate, resveratrol and quercetin enhance the Nrf2-ARE pathway, thereby inducing antioxidant enzymes (44-47). Certain constituents of RVS, including sulfuretin and butein, may stimulate this pathway 
and this has been demonstrated in a human neuronal cell line damaged by amyloid- $\beta$ (48) and a human dental pulp cell line challenged with hydrogen peroxide (49). However, to the best of our knowledge, it has not yet been reported that RVS or its constituents exert therapeutic effects on IRI-mediated AKI by modulating the Nrf2/ARE pathway.

The results of the current study determined that the activation of Nrf2/ARE-mediated antioxidant enzymes was involved in protecting against AKI. The present study indicates that RVS exhibits protective properties against AKI, for example, in the activation of the antioxidant defence system. However, it should be considered that RVS may exert its therapeutic action using alternate mechanisms, including via anti-inflammatory effects. In fact, many factors that may be possible targets of the anti-inflammatory property of RVS are present during the pathogenesis of IRI-induced AKI. During the development of AKI, resident macrophages in the kidney activate and secrete various chemokines and cytokines (50). Furthermore, lymphocytes infiltrate the damaged kidney and contribute to the inflammatory process (51). Thus, owing to its well-known anti-inflammatory effects $(52,53)$, it was assumed that RVS exerts beneficial effects on AKI through this mechanism.

The present study did not determine the upstream machineries of RVS-mediated Nrf2 mobilization, which should be considered a limitation. As aforementioned, Nrf2 is tightly bound to Keap1 and is anchored in the cytoplasm, resulting in its ubiquitination and subsequent degradation under normal conditions. However, when exposed to sub-lethal oxidative damage or treatment with certain pharmaceuticals, the Nrf2-Keap1 protein-protein interaction (PPI) is inhibited, which may liberate Nrf2 from Keap1, leading to its translocation into the nucleus (54-56). Therefore, using individual compounds within RVS, including, GA, fustin, fisetin, sulfuretin and butein and additional approaches, including a binding activity assay for the identification of Nrf2-Keap1 PPI inhibitory roles in the context of AKI are required to establish the precise action of RVS on AKI.

In conclusion, the present study indicated that the intraoral administration of RVS induces a therapeutic effect on IRI-induced AKD. These effects may be due to the attenuation of ROS production via the upregulation of the antioxidant defense system in renal tubular cells. Using the crude extract of RVS, as well as individual compounds within RVS, novel approaches for unveiling multifunctional therapeutic mechanisms and specific molecular targets may be required to determine the protective effects of RVS on AKI.

\section{Acknowledgements}

The current study was supported by the Development of Forest Science and Technology (grant no. S111414L030100) and the Korea Research Foundation (grant nos. NRF-2014R1A1A403005726 and NRF-2016R1C1B2012351).

\section{References}

1. Qiao X, Li RS, Li H, Zhu GZ, Huang XG, Shao S and Bai B Intermedin protects against renal ischemia-reperfusion injury by inhibition of oxidative stress. Am J Physiol Renal Physiol 304: F112-F119, 2013.
2. Lin M, Li L, Li L, Pokhrel G, Qi G, Rong R and Zhu T: The protective effect of baicalin against renal ischemia-reperfusion injury through inhibition of inflammation and apoptosis. BMC Complement Altern Med 14: 19, 2014.

3. Ponticelli C: Ischaemia-reperfusion injury: A major protagonist in kidney transplantation. Nephrol Dial Transplant 29: 1134-1140, 2014.

4. Bonventre JV and Yang L: Cellular pathophysiology of ischemic acute kidney injury. J Clin Invest 121: 4210-4221, 2011.

5. Munshi R, Hsu C and Himmelfarb J: Advances in understanding ischemic acute kidney injury. BMC Med 9: 11, 2011.

6. Yellon DM and Hausenloy DJ: Myocardial reperfusion injury. N Engl J Med 357: 1121-1135, 2007.

7. Walker LM, York JL, Imam SZ, Ali SF, Muldrew KL and Mayeux PR: Oxidative stress and reactive nitrogen species generation during renal ischemia. Toxicol Sci 63: $143-148,2001$.

8. Cutrín JC, Zingaro B, Camandola S, Boveris A, Pompella A and Poli G: Contribution of gamma glutamyl transpeptidase to oxidative damage of ischemic rat kidney. Kidney Int 57: 526-533, 2000.

9. Moss NG, Vogel PA, Kopple TE and Arendshorst WJ: Thromboxane-induced renal vasoconstriction is mediated by the ADP-ribosyl cyclase CD38 and superoxide anion. Am J Physiol Renal Physiol 305: F830-F838, 2013.

10. Ugochukwu NH and Cobourne MK: Modification of renal oxidative stress and lipid peroxidation in streptozotocin-induced diabetic rats treated with extracts from Gongronema latifolium leaves. Clin Chim Acta 336: 73-81, 2003.

11. Kandemir FM, Ozkaraca M, Yildirim BA, Hanedan B, Kirbas A, Kilic K, Aktas E and Benzer F: Rutin attenuates gentamicin-induced renal damage by reducing oxidative stress, inflammation, apoptosis, and autophagy in rats. Renal Failure 37: 518-525, 2015.

12. Aragno M, Cutrin JC, Mastrocola R, Perrelli MG, Restivo F, Poli G, Danni O and Boccuzzi G: Oxidative stress and kidney dysfunction due to ischemia/reperfusion in rat: Attenuation by dehydroepiandrosterone. Kidney Int 64: 836-843, 2003.

13. Ozkok A and Edelstein CL: Pathophysiology of cisplatin-induced acute kidney injury. Biomed Res Int 2014: 967826, 2014

14. Kobayashi A, Kang MI, Watai Y, Tong KI, Shibata T, Uchida K and Yamamoto M: Oxidative and electrophilic stresses activate Nrf2 through inhibition of ubiquitination activity of Keap1. Mol Cell Biol 26: 221-229, 2006.

15. Chen J, Wang W, Zhang Q, Li F, Lei T, Luo D, Zhou H and Yang B: Low molecular weight fucoidan against renal ischemia/reperfusion injury via inhibition of the MAPK signaling pathway. PLoS One 8: e56224, 2013.

16. Zhong Y, Deng Y, Chen Y, Chuang PY and Cijiang He J: Therapeutic use of traditional Chinese herbal medications for chronic kidney diseases. Kidney Int 84: 1108-1118, 2013.

17. Chen W, Jia Z, Pan MH and Anandh Babu PV: Natural products for the prevention of oxidative Stress-related diseases: Mechanisms and strategies. Oxid Med Cell Longev 2016: $4628502,2016$.

18. Su ZY, Shu L, Khor TO, Lee JH, Fuentes F and Kong AN: A perspective on dietary phytochemicals and cancer chemoprevention: Oxidative stress, Nrf2, and epigenomics. Top Curr Chem 329: 133-162, 2013.

19. Kitts DD and Lim KT: Antitumorigenic and cytotoxic properties of an ethanol extract from Rhus verniciflua Stokes (RVS). J Toxicol Environ Health A 64: 357-371, 2001.

20. Kim SA, Kim SH, Kim IS, Lee D, Dong MS, Na CS, Nhiem X and Yoo HH: Simultaneous determination of bioactive phenolic compounds in the stem extract of Rhus verniciflua stokes by high performance liquid chromatography. Food Chem 141: 3813-3819, 2013.

21. Badhani B, Sharma N and Kakkar R: Gallic acid: A versatile antioxidant with promising therapeutic and industrial applications. RSC Advances 5: 27540-27557, 2015.

22. Choi YJ, Do GM, Shin JH, Kim JY and Kwon O: Standardized Rhus verniciflua stokes extract and its major flavonoid fustin attenuate oxidative stress induced by tert-butyl hydroperoxide via activation of nuclear factor erythroid 2-related factor. J Korean Soc Appl Biol Chem 57: 27-30, 2014.

23. Lee DS, Kim KS, Ko W, Li B, Jeong GS, Jang JH, Oh H and Kim YC: The cytoprotective effect of sulfuretin against tert-Butyl hydroperoxide-induced hepatotoxicity through Nrf2/ARE and JNK/ERK MAPK-mediated heme oxygenase-1 expression. Int J Mol Sci 15: 8863-8877, 2014. 
24. Serobatse KRN and Kabanda MM: Antioxidant and antimalarial properties of butein and homobutein based on their ability to chelate iron (II and III) cations: A DFT study in vacuo and in solution. Eur Food Res Technol 242: 71-90, 2016.

25. Kiliç I and Yeşiloğlu Y: Spectroscopic studies on the antioxidant activity of p-coumaric acid. Spectrochim Acta A Mol Biomol Spectrosc 115: 719-724, 2013.

26. Lee SE, Jeong SI, Yang H, Park CS, Jin YH and Park YS: Fisetin induces Nrf2-mediated HO-1 expression through PKC- $\delta$ and $p 38$ in human umbilical vein endothelial cells. J Cell Biochem 112: 2352-2360, 2011

27. Pandurangan AK, Mohebali N, Norhaizan ME and Looi CY: Gallic acid attenuates dextran sulfate sodium-induced experimental colitis in BALB/c mice. Drug Des Devel Ther 9: 3923-3934, 2015

28. Gil MN, Choi DR, Yu KS, Jeong JH, Bak DH, Kim DK, Lee NS, Lee JH, Jeong YG, Na CS, et al: Rhus verniciflua Stokes attenuates cholestatic liver cirrhosis-induced interstitial fibrosis via Smad3 down-regulation and Smad7 up-regulation. Anat Cell Biol 49: 189-198, 2016.

29. Institute for Laboratory Animal Research (ILAR): Guide for the care and use of laboratory animals. National Academy Press, Washington, DC, 2011.

30. Turpaev KT: Reactive oxygen species and regulation of gene expression. Biochemistry (Mosc) 67: 281-292, 2002

31. Day RM and Suzuki YJ: Cell proliferation, reactive oxygen and cellular glutathione. Dose Response 3: 425-442, 2006.

32. Dixit R and Cyr R: Cell damage and reactive oxygen species production induced by fluorescence microscopy: Effect on mitosis and guidelines for non-invasive fluorescence microscopy. Plant J 36: 280-290, 2003.

33. Sauer H, Wartenberg M and Hescheler J: Reactive oxygen species as intracellular messengers during cell growth and differentiation. Cell Physiol Biochem 11: 173-186, 2001

34. Nath KA and Norby SM: Reactive oxygen species and acute renal failure. Am J Med 109: 665-678, 2000.

35. Liou GY and Storz P: Reactive oxygen species in cancer. Free Radic Res 44, 2010, 10.3109/10715761003667554.

36. Mittal M, Siddiqui MR, Tran K, Reddy SP and Malik AB Reactive oxygen species in inflammation and tissue injury. Antioxid Redox Signal 20: 1126-1167, 2014.

37. Pham-Huy LA, He H and Pham-Huy C: Free radicals, antioxidants in disease and health. Int J Biomed Sci 4: 89-96, 2008.

38. Kelly FJ: Use of antioxidants in the prevention and treatment of disease. J Int Fed Clin Chem 10: 21-23, 1998

39. Hu R and Kong AN: Activation of MAP kinases, apoptosis and nutrigenomics of gene expression elicited by dietary cancer-prevention compounds. Nutrition 20: 83-88, 2004.

40. Chang SY, Chen YW, Zhao XP, Chenier I, Tran S, Sauvé A Ingelfinger JR and Zhang SL: Catalase prevents maternal diabetes-induced perinatal programming via the Nrf2-HO-1 defense system. Diabetes 61: 2565-2574, 2012.

41. Motohashi H, Kimura M, Fujita R, Inoue A, Pan X, Takayama M, Katsuoka F, Aburatani H, Bresnick EH and Yamamoto $\mathrm{M}$ NF-E2 domination over Nrf2 promotes ROS accumulation and megakaryocytic maturation. Blood 115: 677-686, 2010.

42. Johnson JA, Johnson DA, Kraft AD, Calkins MJ, Jakel RJ, Vargas MR and Chen PC: The Nrf2-ARE pathway: An indicator and modulator of oxidative stress in neurodegeneration. Ann N Y Acad Sci 1147: 61-69, 2008

43. Petri S, Körner S and Kiaei M: Nrf2/ARE signaling pathway: Key mediator in oxidative stress and potential therapeutic target in ALS. Neurol Res Int 2012: 878030, 2012.
44. Yang $\mathrm{C}$, Zhang $\mathrm{X}$, Fan $\mathrm{H}$ and Liu Y: Curcumin upregulates transcription factor $\mathrm{Nrf} 2, \mathrm{HO}-1$ expression and protects rat brains against focal ischemia. Brain Res 1282: 133-141, 2009.

45. Krajka-Kuźniak V, Szaefer H, Stefański T, Sobiak S, Cichocki M and Baer-Dubowska W: The effect of resveratrol and its methylthio-derivatives on the Nrf2-ARE pathway in mouse epidermis and HaCaT keratinocytes. Cell Mol Biol Lett 19: 500-516, 2014

46. Schadich E, Hlaváč J, Volná T, Varanasi L, Hajdúch M and Džubák P: Effects of ginger phenylpropanoids and quercetin on Nrf2-ARE pathway in human BJ fibroblasts and HaCaT keratinocytes. Biomed Res Int 2016: 2173275, 2016.

47. Sahin K, Tuzcu M, Gencoglu H, Dogukan A, Timurkan M, Sahin N, Aslan A and Kucuk O: Epigallocatechin-3-gallate activates Nrf2/HO-1 signaling pathway in cisplatin-induced nephrotoxicity in rats. Life Sci 87: 240-245, 2010.

48. Kwon SH, Ma SX, Hwang JY, Lee SY and Jang CG: Involvement of the Nrf2/HO-1 signaling pathway in sulfuretin-induced protection against amyloid beta25-35 neurotoxicity. Neuroscience 304: $14-28,2015$

49. Lee DS, Li B, Kim KS, Jeong GS, Kim EC and Kim YC: Butein protects human dental pulp cells from hydrogen peroxide-induced oxidative toxicity via Nrf2 pathway-dependent heme oxygenase-1 expressions. Toxicol In Vitro 27: 874-881, 2013.

50. Ramesh G and Reeves WB: TNF-alpha mediates chemokine and cytokine expression and renal injury in cisplatin nephrotoxicity. J Clin Invest 110: 835-842, 2002.

51. Okyay GU, İnal S, Öneç K, Er RE, Paşaoğlu O, Paşaoğlu H, Derici U and Erten Y: Neutrophil to lymphocyte ratio in evaluation of inflammation in patients with chronic kidney disease. Ren Fail 35: 29-36, 2013

52. Ip WKE, Hoshi N, Shouval DS, Snapper S and Medzhitov R: Anti-inflammatory effect of IL-10 mediated by metabolic reprogramming of macrophages. Science 356: 513-519, 2017.

53. Flaishon L, Hart G, Zelman E, Moussion C, Grabovsky V, Lapidot Tal G, Feigelson S, Margalit R, Harmelin A, Avin-Wittenberg T, et al: Anti-inflammatory effects of an inflammatory chemokine: CCL2 inhibits lymphocyte homing by modulation of CCL21-triggered integrin-mediated adhesions. Blood 112: 5016-5025, 2008.

54. Itoh K, Wakabayashi N, Katoh Y, Ishii T, Igarashi K, Engel JD and Yamamoto M: Keap1 represses nuclear activation of antioxidant responsive elements by Nrf2 through binding to the amino-terminal Neh2 domain. Genes Dev 13: 76-86, 1999.

55. Satoh T, Okamoto SI, Cui J, Watanabe Y, Furuta K, Suzuki M, Tohyama K and Lipton SA: Activation of the Keap1/Nrf2 pathway for neuroprotection by electrophillic (correction of electrophillic) phase II inducers. Proc Natl Acad Sci USA 103: 768-773, 2006.

56. Itoh K, Wakabayashi N, Katoh Y, Ishii T, O'Connor $\mathrm{T}$ and Yamamoto M: Keap1 regulates both cytoplasmic-nuclear shuttling and degradation of Nrf2 in response to electrophiles. Genes Cells 8: 379-391, 2003.

This work is licensed under a Creative Common Attribution-NonCommercial-NoDerivatives 4.0 International (CC BY-NC-ND 4.0) License. 